09

\title{
Определение субпиксельных микросмещений спекл-структуры методом фазового сдвига поля пространственного спектра
}

\author{
(С Л.А. Максимова, ${ }^{1}$ П.В. Рябухо, ${ }^{1,2}$ Н.Ю. Мысина, ${ }^{1}$ В.П. Рябухо ${ }^{1,2}$ \\ ${ }^{1}$ Институт проблем точной механики и управления РАН, \\ 410028 Саратов, Россия \\ ${ }^{2}$ Саратовский национальный исследовательский государственный университет им. Н.Г. Чернышевского, \\ 410012 Саратов, Россия \\ e-mail: MaksimovaLA@yandex.ru
}

(Поступило в Редакцию 8 декабря 2016 г.)

\begin{abstract}
Предложен и экспериментально реализован метод измерения субпиксельных микросмещений спеклструктуры, основанный на определении параметров линейного фазового набега, образующегося в поле комплексного пространственного спектра смещенной спекл-структуры, путем численного введения дополнительного фазового сдвига спектра и корреляционного анализа возникающего смещения линейного фазового набега в пространственном спектре спеклограммы. Метод позволяет расширить диапазон измерений, производимых с помощью метода цифровой спекл-фотографии, для случая, когда период интерференционных полос, формируемых в суммарном пространственном спектре спеклограмм, оказывается существенно больше ширины спектра.
\end{abstract}

DOI: $10.21883 /$ JTF.2017.08.44741.2128

Спекл-структура формируется при отражении когерентного лазерного излучения от рассеивающей поверхности или при прохождении через пространственно случайно неоднородную среду [1,2]. Изменения, происходящие с рассеивающими объектами, вызывают изменения в амплитудно-фазовом распределении комплексной амплитуды и интенсивности спекл-поля. С помощью методов спекл-интерферометрии можно определить изменения, произошедшие в спекл-полях, и, таким образом, получить информацию об изменении состояния рассеивающего объекта $[2,3]$.

Использование в цифровой спекл-интерферометрии $[3,4-6]$ метода взаимной корреляции смещенной и несмещенной спекл-структур позволяет получать информацию о смещении спеклов без преобразования в частотную область. Корреляционные методы позволяют измерять смещения спеклов в изображениях с точностью до периода расположения пикселей матрицы цифровой фотокамеры $[4,5]$, величина которого используется в качестве рабочей меры длины в этих методах. Для измерений микросмещений изображений в субпиксельном диапазоне применяются итерационные вычислительные подходы [6]. В [7] субпиксельная точность корреляционного метода достигается с помощью итерационных подходов и статистического анализа зарегистрированных спекл-полей.

В настоящей работе предложен метод обработки комплексных пространственных спектров изображений спекл-структур для измерения их микросмещений в субпиксельном диапазоне на основе определения параметров линейного фазового набега, образующегося в поле комплексного пространственного спектра смещенной спекл-структуры, путем численного введения дополнительного фазового сдвига спектра и корреляционного анализа возникающего смещения линейного фазового набега в пространственном спектре спеклограммы. Введение дополнительного постоянного фазового сдвига в поле пространственного спектра приводит к соответствующему смещению линейного фазового набега в спектре смещенной спеклограммы. Для определения величины смещения линейного участка графика функции фазового набега используется построчный корреляционный анализ положений точек графика до смещения и после. Предложенный метод позволяет найти величину наклона линейного участка графика функции фазового набега в пределах пространственного спектра и определить с достаточно большой точностью субпиксельное смещение спекл-структуры.

Однородное смещение спекл-структуры $\Delta x$ относительно ее исходного положения вызывает в комплексном пространственном спектре спекл-структуры линейный фазовый набег - фазовую модуляцию, параметры которой (угол наклона линейного участка графика функции фазового набега) зависят от величины взаимного смещения спекл-структур $\Delta x$. Фазовая модуляция проявляется в суммарном пространственном спектре исходной и смещенной спекл-структур - в дифракционном гало в виде системы интерференционных полос [2-4]. Формирование интерференционной картины в дифракционном гало дает возможность визуализировать сигнал и контролировать процесс измерения, если период интерференционных полос меньше размеров гало. Преобразование в частотную область позволяет получить измеряемые величины с субпиксельной точностью по распределению интенсивности интерференционной картины дифракционного гало [8] или определить субпиксельные смещения по наклону линейного участка графика функции фазового набега в поле комплексного пространственного спектра 


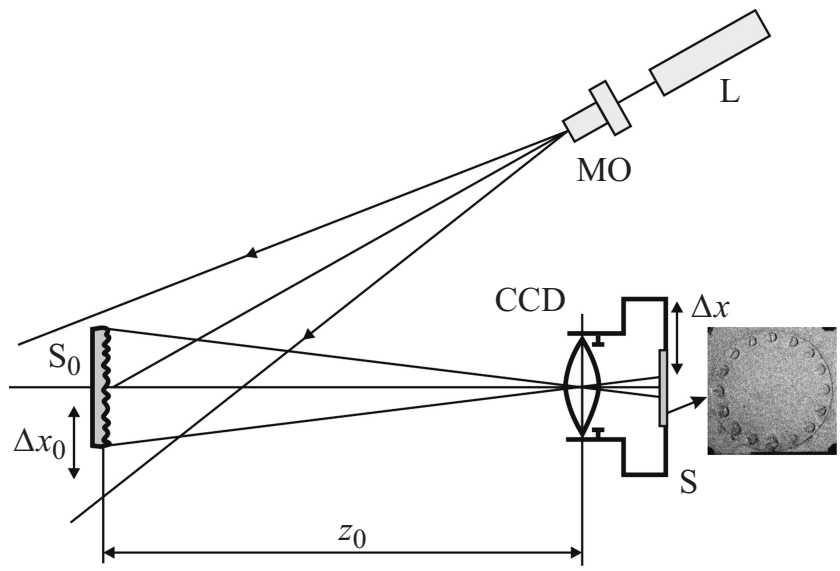

Рис. 1. Оптическая схема записи спекл-структур сфокусированного изображения с использованием цифровой фотокамеры: $\mathrm{L}-$ лазер $(\lambda \approx 0.63 \mu \mathrm{m}), \mathrm{MO}-$ микрообъектив $\left(20^{\times}, N A=0.4\right), \mathrm{S}_{0}-$ объект, $\mathrm{CCD}-$ цифровая фотокамера, $\mathrm{S}$ - спекл-модулированное изображение, регистрируемое на матрице цифровой фотокамеры.

смещенной спекл-структуры [9]. В реальных условиях анализ функции фазового набега затруднен из-за эффектов случайной пространственной модуляции, поэтому в [9] для анализа сигнала используются итерационные и интерполяционные вычислительные процедуры.

На рис. 1 представлена схема реализации метода цифровой спекл-фотографии отражающих рассеивающих объектов с цифровой регистрацией спекл-структуры действительного изображения поверхности объекта. На матрицу цифровой фотокамеры проецируются и затем регистрируются спекл-структуры рассеянного объектом поля, соответствующие исходному состоянию и смещенному в результате поперечного сдвига рассеивающего объекта. В наших экспериментах коэффициент поперечного уменьшения оптической системы (рис. 1) рассчитывался таким образом, чтобы смещение рассеивающего объекта $\Delta x^{\prime}=100 \mu \mathrm{m}$ соответствовало смещению регистрируемого изображения объекта и спекл-структуры в пространстве изображений $\Delta x=1$ pixel $\approx 12.2 \mu \mathrm{m}$, где pixel означает период расположения пикселей. На рис. 2, a представлено изображение по интенсивности разностного пространственного спектра спекл-структур с их относительным смещением $\Delta x=0.4$ pixel.

На рис. 2, $b$ представлены графики функции линейного фазового набега, возникающего в поле комплексного пространственного спектра смещенной спекл-структуры по отношению к несмещенной: исходный график фазового набега 1 и смещенный график из-за дополнительно введенного фазового сдвига $\Delta \phi=-\pi / 20 \mathrm{rad} 2$. Графики на рис. $2, b$ получены путем усреднения по 600 построчным реализациям вдоль оси $\eta$ на линейном участке графиков в пределах дифракционного гало (рис. 2,a). Усреднение необходимо для уменьшения случайной шумовой составляющей, возникающей из-за эффектов некоторой декорреляции спекл-структур, возникающей в оптической системе. Вне области дифракционного гало на пространственное распределение фазового набега оказывают влияние сильные нелинейные модуляции и шумы, в которых теряется информационная составляющая.

Для дискретных распределений комплексной амплитуды в пространственных спектрах исходной и смещенной спекл структур можно записать следующие выражения [10]:

$$
\begin{aligned}
\mathrm{F}\left(I_{0}(x, y)\right) & =\sum_{x=0}^{M-1} \sum_{y=0}^{N-1} I_{0}(x, y) \exp \left[i 2 \pi\left(\frac{x}{M} \xi+\frac{y}{N} \eta\right)\right] \\
& =H_{0}(\xi, \eta), \\
\mathrm{F}\left(I_{S}(x, y)\right) & =\sum_{x=0}^{M-1} \sum_{y=0}^{N-1} I_{0}(x-\Delta x, y) \\
& \times \exp \left[i 2 \pi\left(\frac{x}{M} \xi+\frac{y}{N} \eta\right)\right]=H_{0}(\xi, \eta) \exp \left(i 2 \pi \frac{\Delta x}{M} \xi\right),
\end{aligned}
$$

где $(x, y)$ и $(\xi, \eta)$ - дискретные координаты соответственно в плоскости спеклограммы и в плоскости пространственного спектра, $\mathrm{F}$ - символ пространственного фурье-преобразования, $I_{0}(x, y)$ и $I_{S}(x, y)$ - дискретные распределения интенсивности исходной и смещенной спекл-структур, $H_{0}(\xi, \eta)$ - комплексная амплитуда поля пространственного спектра исходной спекл-структуры, $N \times M-$ размер матрицы в количестве пикселей. Из (1) можно получить выражения для функций фазового набега $\Delta \varphi(\xi)$ (рис. $2, b)$ и определения величины смещения спекл-структуры $\Delta x$ :

$$
\begin{aligned}
& \Delta \varphi_{1}=2 \pi \frac{\Delta x}{M} \xi_{1} \\
& \Delta \varphi_{2}=2 \pi \frac{\Delta x}{M} \xi_{2}+\Delta \phi \\
& \Delta \varphi_{1}=\Delta \varphi_{2}
\end{aligned} \mid \Rightarrow \Delta x=\frac{\Delta \phi M}{2 \pi\left(\xi_{1}-\xi_{2}\right)}=\frac{\Delta \phi M}{2 \pi \Delta \xi} .
$$

Величину $\Delta \xi$ можно определить с помощью построчного (вдоль $\xi$ ) корреляционного анализа взаимного смещения точек графиков функций фазового набега (рис. $2, b$ ) на линейном участке вдоль оси $\xi$. На рис. $3, a$ представлен увеличенный фрагмент расположения точек центральной линейной области графиков (рис. 2,b). Определим для обоих графиков (рис. 2,b) функции $I_{1}\left(\xi, \Delta \varphi_{j}\right)$ и $I_{2}\left(\xi, \Delta \varphi_{j}\right)$, зависящие от $\xi$, при фиксированной ординате $\Delta \varphi_{j}$. Значения этих функций равны единице в точках, принадлежащих графикам, и нулю для остальных точек. Усредненные функции автокорреляции и взаимной корреляции смещения точек вдоль оси $\xi$, согласно свойствам преобразования Фурье [10], можно 

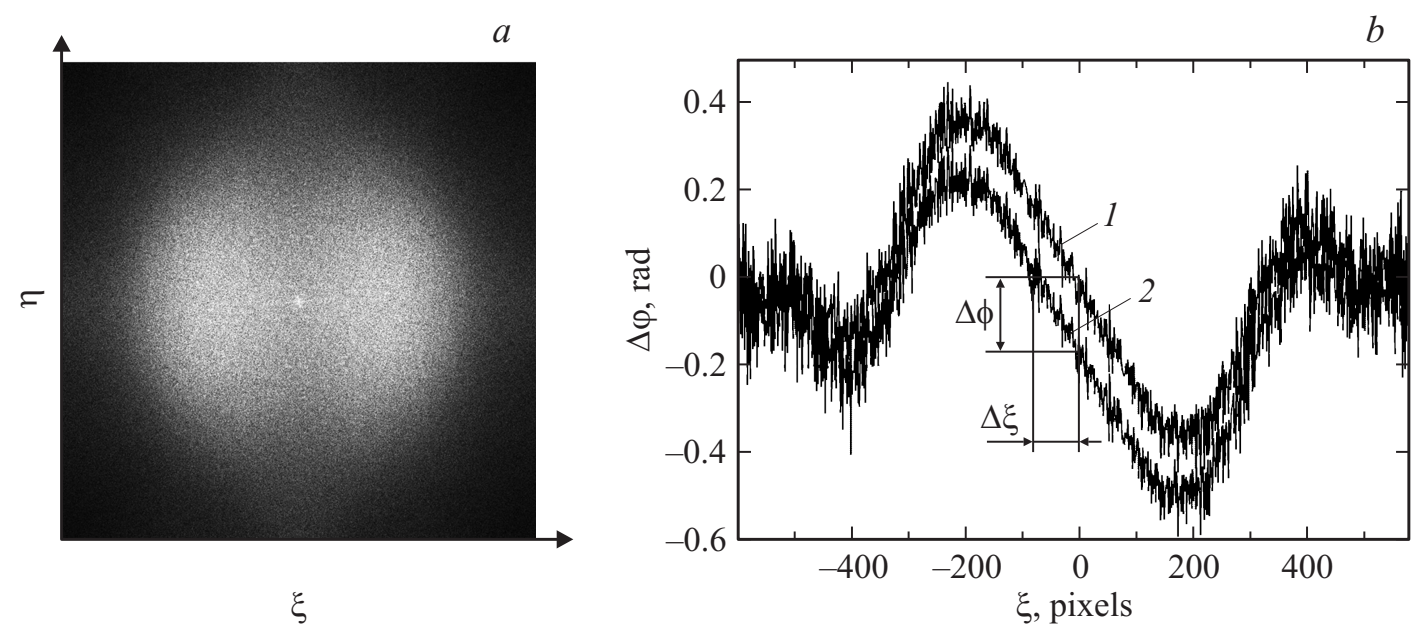

Рис. 2. Разностный пространственный спектр исходной и смещенной спекл-структур на величину $\Delta x=0.4$ pixel $(a)$; графики функции линейного фазового набега в пределах дифракционного гало, возникающего в поле комплексного пространственного спектра смещенной спекл-структуры $(b): 1-$ без фазового сдвига, $2-$ с дополнительным фазовым сдвигом $\Delta \phi=-\pi / 20 \mathrm{rad}$.
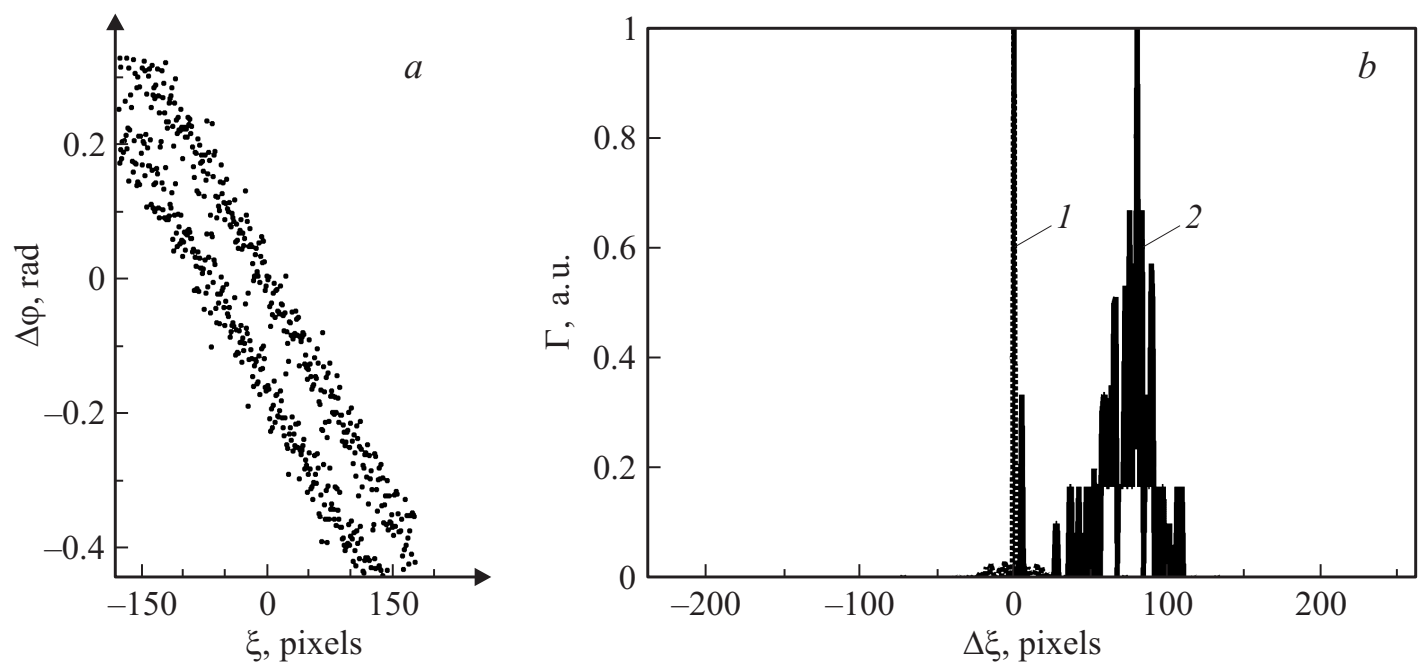

Рис. 3. Точки центральной области графиков фазового набега $(a)$; нормированные усредненные корреляционные функции расположения точек графиков функций фазового набега на линейном участке вдоль оси $\xi(b): 1-$ автокорреляционная функция расположения точек на одном графике, 2 - взаимная корреляционная функция расположения точек на двух графиках.

определить по формулам

$$
\begin{aligned}
& \Gamma_{11}(\Delta \xi)=\sum_{j=1}^{n} \sum_{\xi=0}^{M-1} I_{1}\left(\xi, \Delta \varphi_{j}\right) I_{1}\left(\xi-\Delta \xi, \Delta \varphi_{j}\right) \\
& =\sum_{j=1}^{n} F^{-1}\left\{F\left(I_{1}\left(\xi, \Delta \varphi_{j}\right)\right) \times F^{*}\left\{F\left(I_{1}\left(\xi, \Delta \varphi_{j}\right)\right)\right\},\right. \\
& \Gamma_{12}(\Delta \xi)=\sum_{j=1}^{n} \sum_{\xi=0}^{M-1} I_{1}\left(\xi, \Delta \varphi_{j}\right) I_{2}\left(\xi-\Delta \xi, \Delta \varphi_{j}\right) \\
& =\sum_{j=1}^{n} F^{-1}\left\{F\left(I_{1}\left(\xi, \Delta \varphi_{j}\right)\right) \times F^{*}\left(I_{2}\left(\xi, \Delta \varphi_{j}\right)\right)\right\},
\end{aligned}
$$

где $F$ и $F^{-1}-$ прямое и обратное преобразования Фурье, ${ }^{*}$ - комплексное сопряжение, $n-$ число строк, по которым производится усреднение $n=360$.

На рис. 3, $b$ представлены графики нормированных усредненных корреляционных функций расположения точек функций фазового набега (рис. 3, $a$ ). Величину $\Delta \xi$ можно определить по смещению центрального максимума функции взаимной корреляции $\Gamma_{12}(\Delta \xi)$ относительно максимума автокорреляционной функции $\Gamma_{11}(\Delta \xi)$ (рис. $3, b$ ). В частности, в нашем эксперименте для графиков на рис. $2, b$ была получена величина взаимного смещения графиков вдоль оси $\xi \Delta \xi \approx 79$ pixel и, учитывая $M=1280$ pixel, по формуле (2) можно определить величину смещения спекл-структуры $\Delta x \approx 0.405$ pixel.

Абсолютную погрешность определения величины смещения спекл-структуры можно получить как раз- 
ность между заданным значением 0.4 pixel и, полученным в результате обработки фазовых набегов, $\delta\left(\Delta x_{p}\right) \approx 0.005$ pixel. С помощью корреляционного метода величину $\Delta \xi$ можно определить с точностью до одного пикселя, $\delta(\Delta \xi)=1$ pixel, тогда абсолютную погрешность $\Delta x$ можно найти, исходя из формулы (2), $\delta\left(\Delta x_{p}\right) \approx\left(\Delta \phi M / 2 \pi(\Delta \xi)^{2}\right) \delta(\Delta \xi) \approx 0.005$ pixel. Расчетная и полученная в эксперименте погрешности совпадают с хорошей точностью.

Проведение измерений с субпиксельной точностью или в субпиксельном диапазоне при реализации метода цифровой спекл-фотографии, как показывают наши эксперименты, возможно при выполнении численных преобразований спекл-структур в область их пространственных частот. Такая точность достигается на основе определения величин параметров линейного фазового набега, возникающего в поле комплексного пространственного спектра смещенной спекл-структуры, с помощью корреляционного анализа смещения линейного фазового набега, образующегося в пространственном спектре спеклограммы при численном введении дополнительного постоянного фазового сдвига спектра. Чем меньше размеры регистрируемых спеклов, тем больше размер дифракционного гало - шире пространственный спектр, и соответственно больше линейный участок функции фазового набега и, следовательно, выше точность выполняемых измерений.

Предложенный в работе метод реализации интерференционных измерений позволяет определять смещение спекл-структуры в субпиксельном диапазоне и с субпиксельной точностью. Это расширяет диапазон измерений, производимых с помощью метода цифровой спеклинтерферометрии, в сторону малых величин смещений и может быть эффективно использовано в практике измерения малых смещений и деформаций рассеивающих объектов.

\section{Список литературы}

[1] Goodman J.W. Speckle Phenomena in Optics: Theory and Applications. Roberts \& Company, Publishers, Englewood, CO, 2006. 387 p.

[2] Osten $W$. (ed.) Optical inspection of microsystems (Optical Science and Engineering). Taylor \& Francis Group, 2007. $503 \mathrm{p}$.

[3] Schnars U., Falldorf C., Watson J., Jueptner W. Digital Holography and Wavefront Sensing. Berlin, Heidelberg: Springer Verlag, 2015. $226 \mathrm{p}$.

[4] Mudassar A.A., Butt S. // Appl. Opt. 2014. Vol. 53. N 5. P. 960-970.

[5] Cofaru C., Philips W., Van Paepegem W. // Opt. Expres. 2013. Vol. 21. N 24. P. 29979-29999.

[6] Grebenyuk A.A., Ryabukho V.P. // Proc. of SPIE. 2011. Vol. 7999. P. 79990B.

[7] Фомин Н.Ф., Мелеева О.В. // Автометрия. 2012. Т. 48. № 3. C. $82-89$.
[8] Максимова Л.А., Рябухо П.В., Мысина Н.Ю., Рябухо В.П. // Изв. Саратовского ун-та. Новая серия. Серия Физика. 2015. Т. 15. Вып. 3. С. 5-11.

[9] Lu H., Huang C., Wang C., Wang X., Fu H., Chen Z. // Appl. Opt. 2014. Vol. 13. P. 2806-2814.

[10] Локшин Г.Р. Основы радиооптики. М.: Интеллект, 2009. $344 \mathrm{c}$. 